\title{
The thyrotropin receptor autoantigen in Graves disease is the culprit as well as the victim
}

\author{
Chun-Rong Chen, ${ }^{1}$ Pavel Pichurin, ${ }^{1}$ Yuji Nagayama, ${ }^{2}$ Francesco Latrofa, ${ }^{1}$ \\ Basil Rapoport, ${ }^{1}$ and Sandra M. McLachlan ${ }^{1}$ \\ ${ }^{1}$ Autoimmune Disease Unit, Cedars-Sinai Research Institute and School of Medicine, University of California, Los Angeles, \\ Los Angeles, California, USA \\ ${ }^{2}$ Department of Pharmacology 1, Nagasaki University School of Medicine, Nagasaki, Japan
}

\begin{abstract}
Graves disease, a common organ-specific autoimmune disease affecting humans, differs from all other autoimmune diseases in being associated with target organ hyperfunction rather than organ damage. Clinical thyrotoxicosis is directly caused by autoantibodies that activate the thyrotropin receptor (TSHR). The etiology of Graves disease is multifactorial, with nongenetic factors playing an important role. Of the latter, there is the intriguing possibility that the molecular structure of the target antigen contributes to the development of thyroid-stimulatory autoantibodies (TSAb's). Among the glycoprotein hormone receptors, only the TSHR undergoes intramolecular cleavage into disulfide-linked subunits with consequent shedding of some of the extracellular, autoantibody-binding A subunits. Functional autoantibodies do not arise to the noncleaving glycoprotein hormone receptors. Recently, TSAb's were found to preferentially recognize shed, rather than attached, A subunits. Here we use a new adenovirus-mediated animal model of Graves disease to show that goiter and hyperthyroidism occur to a much greater extent when the adenovirus expresses the free A subunit as opposed to a genetically modified TSHR that cleaves minimally into subunits. These data show that shed A subunits induce or amplify the immune response leading to hyperthyroidism and provide new insight into the etiology of Graves disease.
\end{abstract}

J. Clin. Invest. 111:1897-1904 (2003). doi:10.1172/JCI200317069.

\section{Introduction}

Graves disease is a common organ-specific autoimmune disease with an incidence of approximately 4 in 10,000 people per year (1). A syndrome including goiter and thyrotoxicosis, the disease differs from all other autoimmune diseases in being associated with target organ hyperfunction rather than organ damage. Thyrotoxicosis is directly caused by autoantibodies that activate the thyrotropin (TSH) receptor (TSHR) (reviewed in ref. 2). Genetic factors play an important role in Graves disease (for example, see ref. 3). A concordance rate of 0.35 between identical twins (4), however, indicates that a substantial contribution derives from nongenetic factors. Of the latter, there is the intriguing possibility that the molecular structure of the target antigen contributes to the development of thyroid-stimulatory autoantibodies (TSAb's). The

Received for publication October 4, 2002, and accepted in revised form April 15, 2003.

Address correspondence to: Sandra McLachlan, Cedars-Sinai Medical Center, 8700 Beverly Boulevard, Suite B-131, Los Angeles, California 90048, USA. Phone: (310) 423-7680; Fax: (310) 423-0221; E-mail: mclachlans@cshs.org.

Conflict of interest: The authors have declared that no conflict of interest exists.

Nonstandard abbreviations used: thyrotropin (TSH); thyrotropin receptor (TSHR); thyroid-stimulatory autoantibody (TSAb); TSH receptor variant encoding free A subunits (TSHR289); modified TSHR unable to cleave into subunits (TSHRD1NET); TSHR Ab that blocks stimulation by TSH (TBAb); thyroxine (T4); TSH-binding inhibition assay (TBI).
TSHR is unique among the glycoprotein hormone receptors in undergoing intramolecular cleavage into disulfide-linked subunits (Figure 1a) $(5,6)$. A consequence of TSHR cleavage is shedding of some of the extracellular, autoantibody-binding A subunits $(7,8)$. Although the physiological relevance of TSHR cleavage is not known, it is noteworthy that functional autoantibodies do not arise to the noncleaving glycoprotein hormone receptors.

Recently, we observed that the epitope for functionally important TSAb's in Graves disease is partially obscured in the WT TSHR but is exposed on the TSHR ectodomain tethered to the cell surface by a glycosylphosphatidylinositol anchor (9). These findings raised the possibility that the shed A subunit, not the full-length TSHR expressed on the cell surface, initiates or enhances the immune response to the TSHR that leads to hyperthyroidism. Testing this hypothesis requires an animal model of Graves disease. No spontaneous model exists, and immunization with TSHR protein plus adjuvant does not replicate the disease (reviewed in ref. 2). In contrast, processing and presentation of TSHR expressed in vivo induces Ab's to the TSHR epitope(s) necessary for receptor activation (10-13).

One of these approaches, intramuscular injection into mice of adenovirus particles containing the cDNA for the human TSHR (13), provides an ideal system for testing the above hypothesis. We constructed adenoviruses encoding either free A subunits (Figure 1c) or 
TSHR engineered to not cleave into subunits (TSHRD1NET; Figure 1b). The data obtained confirm the hypothesis and further our understanding of the pathogenesis of Graves disease.

\section{Methods}

Adenovirus constructs and immunization of mice. The cDNA for TSHR-289 (14) and TSHR-D1NET (15) in the vector pECE-neo were digested with EcoRI (New England Biolabs Inc., Beverly, Massachusetts, USA), blunted with DNA polymerase Klenow fragment (USB Corp., Cleveland, Ohio, USA), digested with XbaI (New England Biolabs Inc.), and ligated into the transfer vector pHMCMV6 (16). Insert-positive plasmids were digested with I-CeuI and PI-SceI (both from BD Biosciences Clontech, Palo Alto, California, USA) and ligated into the same sites of pAdHM4CMV (17). Adenoviruses containing TSHR-289 or TSHRD1NET were linearized with PacI (New England Biolabs Inc.) and transfected into human embryonal kidney 293 cells (HEK293; American Type Culture Collection, Manassas, Virginia, USA) with SuperFect (QIAGEN Inc., Valencia, California, USA). Construction of adenovirus containing the WT TSHR cDNA was described previously (13). TSHR-expressing adenoviruses (Ad-TSHR-WT, Ad-TSHR-289, and AdTSHR-D1NET) and control adenovirus expressing $\beta$-gal (Ad- $\beta$-gal) were propagated in HEK293 cells, purified by $\mathrm{CsCl}$ density-gradient centrifugation, and viral particle concentration determined by measuring the absorbance at $260 \mathrm{~nm}$ (18).

Female BALB/c mice (6-7 weeks old; The Jackson Laboratory, Bar Harbor, Maine, USA) were injected intramuscularly with TSHR-expressing adenovirus or with control Ad- $\beta$-gal $\left(10^{11}\right.$ particles in $50 \mu$ phosphatebuffered saline). Mice were injected three times at three weekly intervals, and blood was drawn 1 week after the second injection. Animals were euthanized 8 weeks (first experiment) or 4 weeks (second experiment) after the third injection to obtain blood and thyroid glands. All animal studies were approved by the Institutional Animal Care and Use Committee and performed with the highest standards of animal care in a pathogen-free facility.

Serum thyroxine levels. Total thyroxine (T4) in mouse sera was measured in undiluted serum $(25 \mu \mathrm{l})$ by radioimmunoassay using a kit (Diagnostic Products Corp., Los Angeles, California, USA).

Thyroid size and histology. Thyroids were fixed in buffered paraformaldehyde ( $\mathrm{pH} 7.4$ ), and 16-18 serial sections obtained for each gland were stained with hematoxylin and eosin. Two-dimensional sizes of individual thyroids were determined by planimetry using the University of Texas Health Science Center San Antonio Image Tool 3.0 (downloadable at http://ddsdx.uthscsa.edu/dig/itdesc. $\mathrm{html}$ ). The values reported (in square millimeters) are for the section with the greatest surface area at the center plane of the three-dimensional structure.

TSHR Ab's measured by ELISA. TSHR Ab's were measured as described previously (19) using ELISA wells coated with purified TSHR-289 $(1 \mu \mathrm{g} / \mathrm{ml}$ in $10 \mathrm{mM}$ Tris, $\mathrm{pH} 7.4,50 \mathrm{mM} \mathrm{NaCl}$ ). TSHR-289 is a variant of the receptor expressed in $\mathrm{CHO}$ cells that corresponds to the extracellular A subunit (20). Duplicate aliquots of sera diluted $1: 10^{3}$ were analyzed and $\mathrm{Ab}$ binding was detected with horseradish peroxidase-conjugated mouse anti-IgG (Sigma-Aldrich, St. Louis, Missouri, USA). The signal was developed with $o$-phenylenediamine and $\mathrm{H}_{2} \mathrm{O}_{2}$, and $\mathrm{OD}$ was read at $490 \mathrm{~nm}$.

TSH-binding inbibition assay. TSHR Ab inhibition of TSH binding to the TSHR was measured using commercial kits according to the protocol of the manufacturer (Kronus Inc., Boise, Idaho, USA). In brief, duplicate serum aliquots $(50 \mu \mathrm{l}$, undiluted in most studies; when indicated, diluted 1:10 in normal mouse serum) were incubated with detergent-solubilized TSHR; ${ }^{125}$ I-TSH was added, and the TSHR Ab a Wild-type TSHR (cleaves)

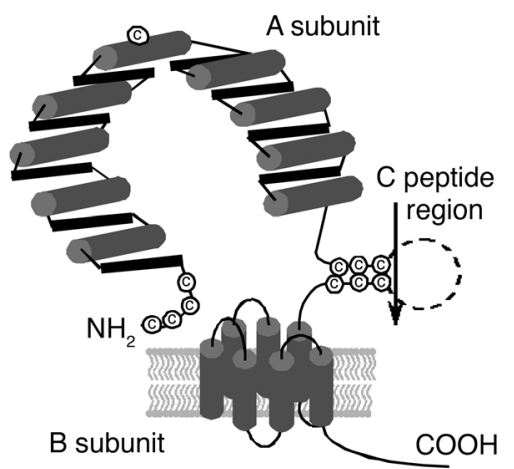

b TSHR-D1NET (noncleaving)

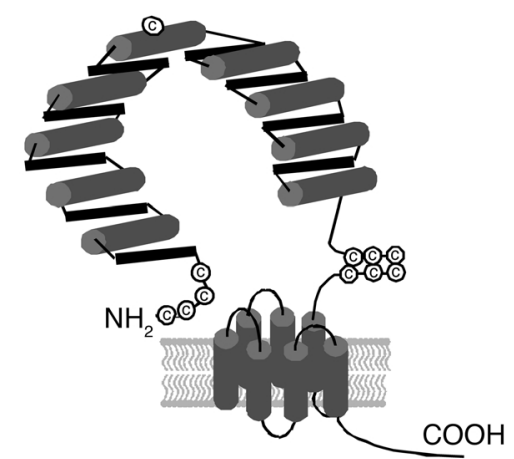

C TSHR-289 (shed A subunit)

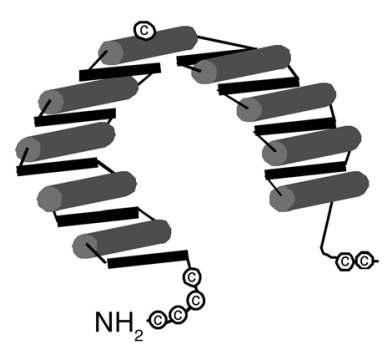

Figure 1

(a) Schematic representation of the WT TSHR that undergoes cleavage into an extracellular A subunit and a serpentine, membrane-spanning B subunit. The cleavage process involves excision of a C peptide region (reviewed in ref. 2). (b) TSHR modified by deletion of amino acid residues 317-366 and substitution of GQE367-369NET. This receptor (TSHR-D1NET) undergoes minimal cleavage into subunits (15). (c) TSHR-289 is the TSHR truncated after amino acid 289, at the approximate region of spontaneous intramolecular cleavage into subunits (14). Consequently, TSHR-289 approximates the free, or shed, A subunit. (Adapted from ref. 9.) 
complexes were precipitated with polyethylene glycol. The TSH-binding inhibition assay (TBI) values were calculated from the formula:

\section{Equation 1}

$$
\left[1-\frac{\text { (TSH binding in test serum }- \text { nonspecific binding })}{\text { (TSH binding in control serum }- \text { nonspecific binding })}\right] \times 100
$$

TSAb and TSH-blocking Ab. TSAb and TSH-blocking $\mathrm{Ab}$ assays cannot be performed under optimal conditions because insufficient serum is available to permit purification of IgG, a step routinely performed using human sera. Instead, we used the standard approach for studying murine Graves disease, namely dilution of whole serum $(13,21)$.

TSAb was measured as described previously (22). Briefly, monolayers of TSHR-expressing $\mathrm{CHO}$ cells (23) in 96-well plates were incubated with test sera (duplicate wells with $3 \mu \mathrm{l}$ serum in $100 \mu \mathrm{l}$ Hank's buffer without $\mathrm{NaCl}$ supplemented with $20 \mathrm{mM}$ Hepes, pH 7.4, $1 \mathrm{mM}$ isobutylmethylxanthine, 220 $\mathrm{mM}$ sucrose, and $0.3 \% \mathrm{BSA})$. After 3 hours incubation $\left(37^{\circ} \mathrm{C}, 5 \% \mathrm{CO}_{2}\right)$, plates were frozen $\left(-80^{\circ} \mathrm{C}\right)$, thawed, supernatants diluted 1:400, and acetylated (20 $\mu \mathrm{l}$ triethylamine and $10 \mu \mathrm{l}$ acetic anhydride $/ \mathrm{ml})$. Total cAMP was measured by radioimmunoassay using cAMP, 2'-O-succinyl-[125I]idotyrosine methyl ester (PE Applied Biosystems, Foster City, California, USA), and a rabbit anti-cAMP Ab (Fitzgerald Industries International Inc., Concord, Massachusetts, USA). Results are expressed as percentage of basal cAMP generated in the presence of serum from normal, untreated mice. As controls in these assays, two
Graves' sera gave TSAb's of $1159 \%$ and $1115 \%$ of normal human serum values.

For detecting TSH-blocking $\mathrm{Ab}$ (TBAb) activity, bovine TSH $(0.2 \mathrm{mU} / \mathrm{ml}$; Sigma-Aldrich, St. Louis, Missouri, USA) was added to the same modified Hanks' buffer. TBAb (percentage of inhibition of TSH-induced cAMP) was calculated as follows:

\section{Equation 2}

$$
\left[1-\frac{\text { (test serum with bTSH }- \text { test serum without bTSH) }}{(\text { normal serum with bTSH }- \text { normal serum without bTSH) }}\right] \times 100
$$

Statistical analyses. A Fisher exact test was used to determine the significance of differences between the number of mice in a group positive or negative for a particular parameter. Significant differences between the magnitudes of responses between mice in different groups were determined by Mann Whitney rank sum tests or, when normally distributed, Student $t$ tests.

\section{Results}

Hyperthyroidism and goiter in mice immunized with different TSHR structural forms. BALB/c mice were injected intramuscularly with adenoviruses expressing different structural forms of the TSHR (Figure 1): the free A subunit (Ad-TSHR-289) or a TSHR that cleaves minimally into A and B subunits (Ad-TSHR-D1NET). For controls, we also immunized mice with adenoviruses expressing $\beta$-gal (Ad- $\beta$-gal) or the WT TSHR (Ad-TSHR-WT), which undergoes partial intramolecular cleavage into $A$ and $B$ subunits.

A cardinal feature of Graves disease is elevated thyroid hormone levels (thyrotoxicosis) consequent to

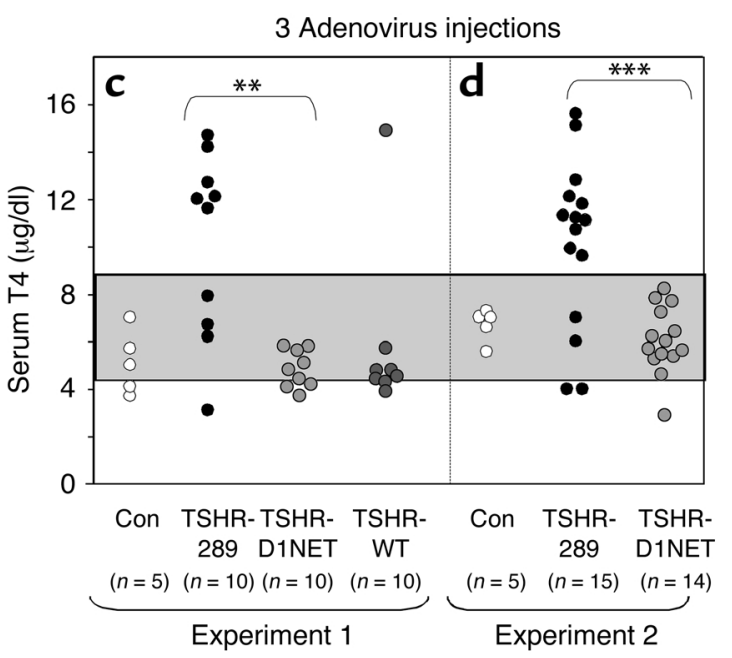

Figure 2

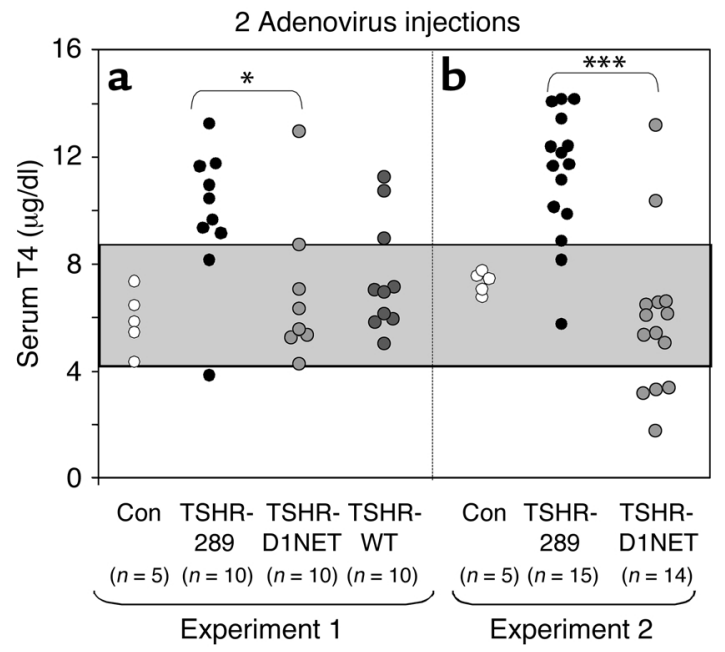

Serum T4 in mice immunized with adenovirus expressing different structural forms of the TSHR. BALB/c mice were immunized with adenovirus expressing the shed A subunit (TSHR-289) and the noncleaving receptor (TSHR-D1NET). As controls, mice were also injected with adenovirus expressing the WTTSHR (TSHR-WT) or $\beta$-gal (Con). Data shown are the individual values for mice in each group (number shown in parentheses). The shaded area indicates the mean \pm 2 SD of values for 15 control adenovirus-immunized mice. Serum T4 levels were measured in mice after two adenovirus injections ( $\mathbf{a}$ and $\mathbf{b}$ ) and at euthanasia after the third adenovirus injections (c and $\mathbf{d})$. Immunization with TSHR-289 adenovirus increased serum T4 levels in more mice than immunization with TSHR-D1NET adenovirus. ${ }^{*} P=0.005 ;{ }^{*} P=0.002$; ***P 0.001 (Fisher exact test). 


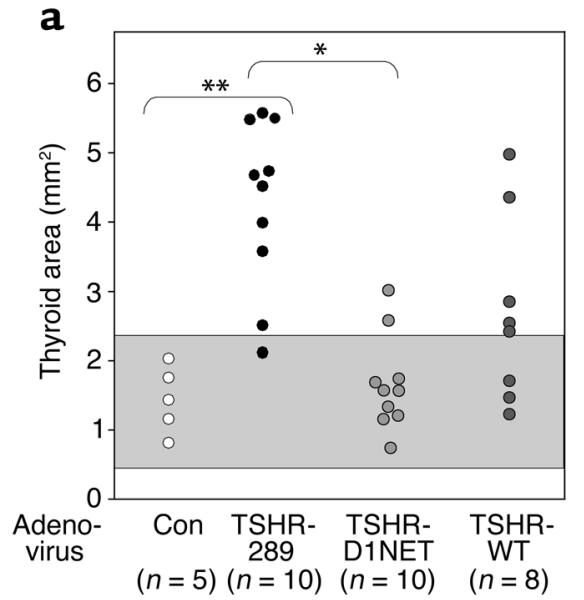

b Control-adenovirus

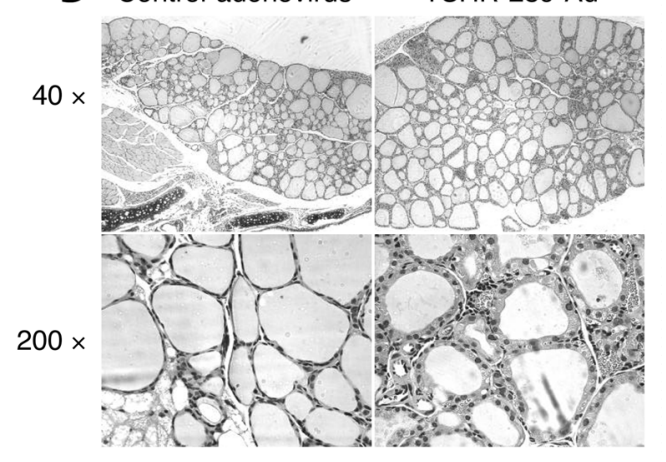

thyroid overactivity (hyperthyroidism), usually associated with goiter. We therefore determined serum T4 levels and thyroid sizes in the mice injected with the different TSHR-expressing adenoviruses. In the first experiment, 1 week after the second immunization with the A subunit-containing virus (Ad-TSHR-289), eight of ten mice had elevated serum $\mathrm{T} 4$ levels relative to control adenovirus-immunized mice (Figure 2a). In contrast, only one of ten mice injected with the noncleaving TSHR (Ad-TSHR-D1NET) was hyperthyroid ( $P=0.005$ vs. Ad-TSHR-289, Fisher exact test). Of the WT TSHR-immunized mice, three of ten were thyrotoxic $(P=0.02$ vs. Ad-TSHR-289). Similar observations were made in a second experiment. Again, there was a much higher incidence of thyrotoxicosis in mice injected with Ad-TSHR-289 than in mice injected with Ad-TSHR-D1NET (Figure 2b; 13 of 15 vs. 2 of 14 mice; $P<0.001$ ).

Serum T4 levels were measured again at euthanasia after the third immunization. The general trend was a decline in T4 from their earlier levels. Indeed, in the first experiment, all TSHR-D1NET-immunized mice, and all but one WT TSHR-immunized mice, were euthyroid (Figure 2c). In the Ad-TSHR-289-immunized group, however, T4 levels were maintained or rose to higher levels in the majority (six of ten) mice. The same pattern was observed in the second experiment; none of the 14 Ad-D1NET mice had elevated T4 levels, whereas 11 of 15 Ad-TSHR-289-immunized mice remained thyrotoxic (Figure $2 \mathrm{~d}, P<0.001$ ).

\section{Figure 3}

Goiter and histology in TSHR-adenovirus-injected mice. (a) Thyroid lobe areas measured by planimetry in mice immunized with adenoviruses expressing TSHR-289, TSHR-D1NET, TSHR-WT, and control adenovirus (Con). The gray area indicates the mean \pm 2 SD of values for control mice. The number of animals with goiters was significantly higher in mice injected with adenovirus TSHR-289 versus control (Con) adenovirus ( $\left.{ }^{*} P=0.002\right)$ and in adenovirus TSHR-289 versus adenovirus TSHR-D1NET-injected mice $\left({ }^{*} P=0.005\right)$ (Fisher exact test). (b) Thyroid histology in representative mice from the control adenovirus (euthyroid animal) and TSHR-289-Ad (hyperthyroid animal) groups. Entire thyroids cannot be shown because the $\times 40$ magnification was the lowest possible with this microscope. Thyroid epithelial morphology is evident at higher magnification $(\times 200)$. Thyrocytes in the adenovirus-TSHR-289-immunized animal are more columnar and vacuolated, consistent with hyperthyroidism.

Thyroids from the great majority of Ad-TSHR-289immunized mice were visibly enlarged, unlike the normal-sized glands in Ad-D1NET-injected mice. Because this visual evaluation is subjective, however, we prepared thyroid sections for histology (see below) and measured thyroid sizes by computerized planimetry in animals from the first experiment. The most marked goiters occurred in the Ad-TSHR-289-immunized mice, with a mean thyroid lobe area of $4.27 \pm 0.39 \mathrm{~mm}^{2}( \pm \mathrm{SEM})$ versus a mean area of $1.44 \pm 0.21 \mathrm{~mm}^{2}$ in the control adenovirus-injected mice $(P<0.001$, Student $t$ test $)$ (Figure 3a). Overall, nine of ten Ad-TSHR-289-immunized mice had thyroid sizes more than $2 \mathrm{SD}$ above the mean value for the control mice. In contrast, only two of ten mice immunized with the vector expressing the noncleaving TSHR

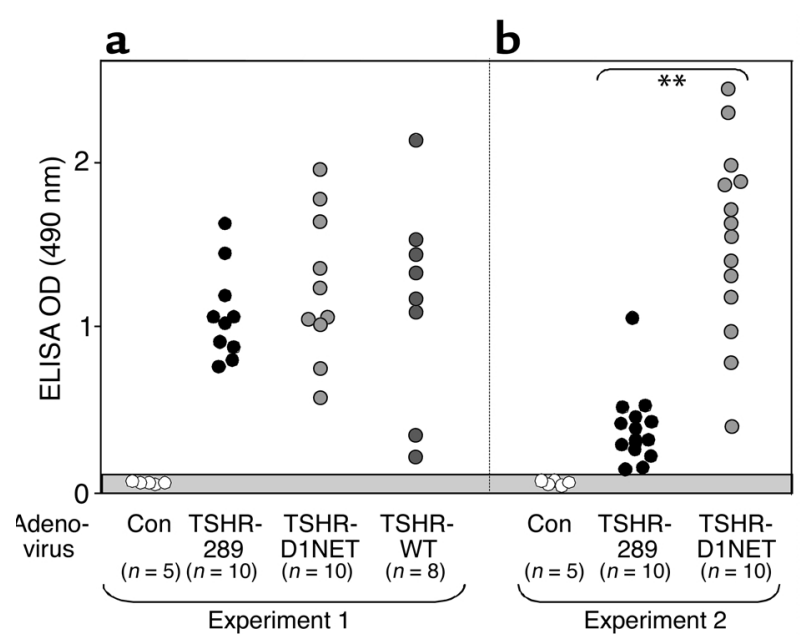

\section{Figure 4}

TSHR Ab's measured by ELISA in mice immunized with TSHR-adenovirus. BALB/c mice were injected intramuscularly with adenovirus expressing the shed A subunit (TSHR-289), the noncleaving receptor (TSHR-D1NET), the WT TSHR (TSHR-WT), or control adenovirus (Con). Ab binding to TSHR protein-coated ELISA wells was tested in sera diluted $1: 10^{3}$ for mice immunized with TSHR-adenovirus and in sera diluted $1: 10^{2}$ for mice injected with control adenovirus. Data shown are the OD values for individual mice in experiment 1 (a) and experiment 2 (b), with the number of animals in each group in parentheses. The gray area indicates the mean \pm 2 SD of values for ten control adenovirus-immunized mice. 


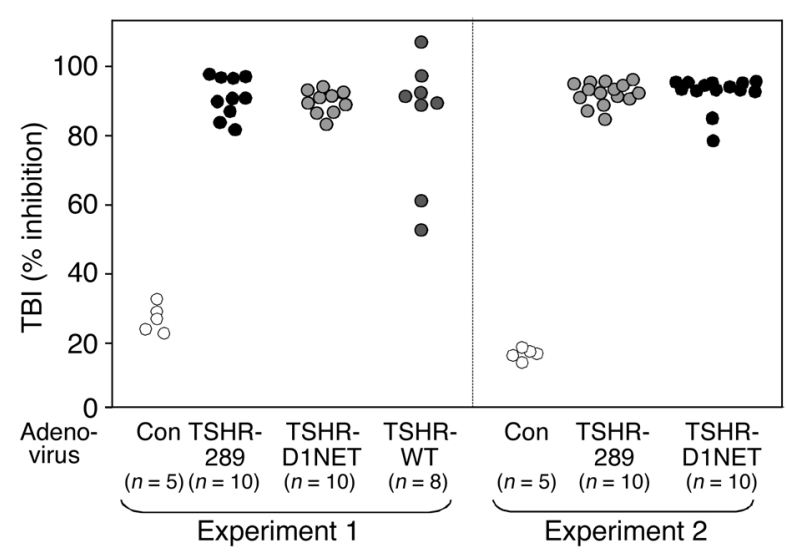

Figure 5

Comparable high levels of TBI immunoglobulins are induced in mice immunized with adenovirus expressing different structural forms of the TSHR. BALB/c mice were injected intramuscularly with adenovirus expressing the shed A subunit (TSHR-289), the noncleaving receptor (TSHR-D1NET), the WT receptor (TSHR-WT), and control adenovirus (Con). TBI values in mice euthanized after the third adenovirus injection are shown for individual animals; the number of mice in each group is shown in parentheses. There were no statistically significant differences in TBI values between the groups of mice immunized with the different TSHR adenovirus constructs.

had significantly increased thyroid sizes $(P<0.002$, Fisher exact test). Thyroid sizes in the WT TSHR-immunized mice were intermediate to the Ad-TSHR-289- and AdTSHR-D1NET-immunized mice. It is noteworthy that this intermediate incidence of goiter in the WT TSHRimmunized mice (four of eight) (Figure 3a) correlated more closely with the incidence of thyrotoxicosis after the second immunization than at the time of euthanasia (Figure 2a). These data are consistent with goiter being a "lagging indicator," taking a longer period of time to revert to normal after a decline in thyroid stimulation and a reversion to euthyroidism in some animals. In general, thyroid lobe sizes correlated well with the serum T4 measurements at euthanasia $(r=0.777 ; P<0.001)$.

Histological examination confirmed that the basis for goiter development was follicular hyperactivity with hyperplasia (Figure 3b). Thyroids were diffusely enlarged, without interstitial lymphocytic infiltration, and there was no nodular progression. As compared with control adenovirus-immunized animals, thyroid follicles in the goiters had cuboidal thyroid epithelial cells with marked intracellular vacuolation, indicating higher secretory activity.

Quantitation of TSHR Ab levels in mice immunized with TSHR adenovirus. One possible explanation for significantly more hyperthyroidism and goiter in mice immunized with Ad-TSHR-289 compared with Ad-D1NET is higher TSHR Ab levels in the former versus the latter group. We examined this possibility in two ways. In the first approach, we quantitated TSHR Ab levels by ELISA in sera from mice euthanized after three adenovirus injections. Sera from animals injected with control adenovirus (diluted 1:100) had only low background values.
In contrast, all mice injected with TSHR-containing adenoviruses developed TSHR Ab's detectable at 1:1,000. TSHR Ab levels were not higher in the Ad-TSHR289-immunized mice than in the Ad-TSHR-D1NET- or Ad-WT-TSHR-immunized mice (Figure 4a). Indeed, in a second experiment, mice immunized with Ad-TSHR-289 had even lower levels than mice injected with Ad-D1-NET (Figure 4b; $P<0.001$, Mann-Whitney rank sum test).

Ab's detected by ELISA could be nonfunctional. We therefore studied the same sera using the TBI assay, which determines epitopic overlap between TSHR Ab's and the physiological ligand (TSH). As for the TSHR Ab ELISA data, all TSHR-immunized animals in both experiment 1 and experiment 2 had high TBI levels regardless of the structural form of the receptor in the adenovirus vector (Figure 5). Most important, there was no difference in TBI levels between the Ad-TSHR289- and the Ad-TSHR-D1NET-immunized animals, despite the greater incidence of thyrotoxicosis and goiter in the former group.

The very high TBI values (>90\% inhibition) suggested that we were operating at the maximum level in the TBI assay using neat serum. We therefore repeated the assay with available sera (second experiment) after dilution (1:10). Sera from both groups of TSHR adenovirus-immunized mice remained TBI positive albeit with, as expected, lower values. Importantly, even after dilution, TBI values were not significantly higher in the Ad-TSHR-289 group (15 mice) than in the Ad-TSHRD1NET group (14 mice) (means \pm SEM of $65 \% \pm 3.5 \%$ and $74.5 \% \pm 4.3 \%$, respectively).

TSA $A$ and TSH-blocking Ab's in adenovirus-immunized mice. Functional TSHR Ab activity in serum reflects the net effect of TSAb's and Ab's that block thyroid activation by TSH or TSAb (TBAb). We tested the mouse sera for both activities in bioassays using the cAMP response in CHO cells expressing the WT TSHR.

After two injections, TSAb activity was significantly higher in the Ad-TSHR-289 group than in the AdTSHR-D1NET group (Figure 6a; $P=0.002$, experiment 1 ; $P<0.002$, experiment 2 ; Mann-Whitney rank sum test). Dramatically, the reciprocal relationship was observed for TBAb (Figure 6b). The majority of sera from almost all mice immunized with the noncleaving TSHR completely blocked TSH action. This TBAb activity was significantly lower in Ad-TSHR-289-injected animals $(P<0.001$ for both experiments). The WT TSHR, which undergoes variable and incomplete cleavage into subunits, had TSAb and TBAb levels intermediate to those in the A subunit and noncleaving TSHR-immunized mice. The divergent patterns for TSAb and TBAb activities persisted after the third and final immunization (Figure 6, $c$ and d).

Taken together, these data indicate that, as in human Graves disease, the TSAb assay by itself provides an imperfect indicator of thyroid overactivity. The most striking observation was the combined importance of TBAb and TSAb activities (discussed below). Thus, the Ad-TSH-289-immunized animals that had the largest goiters and greatest degree of 
2 Adenovirus injections
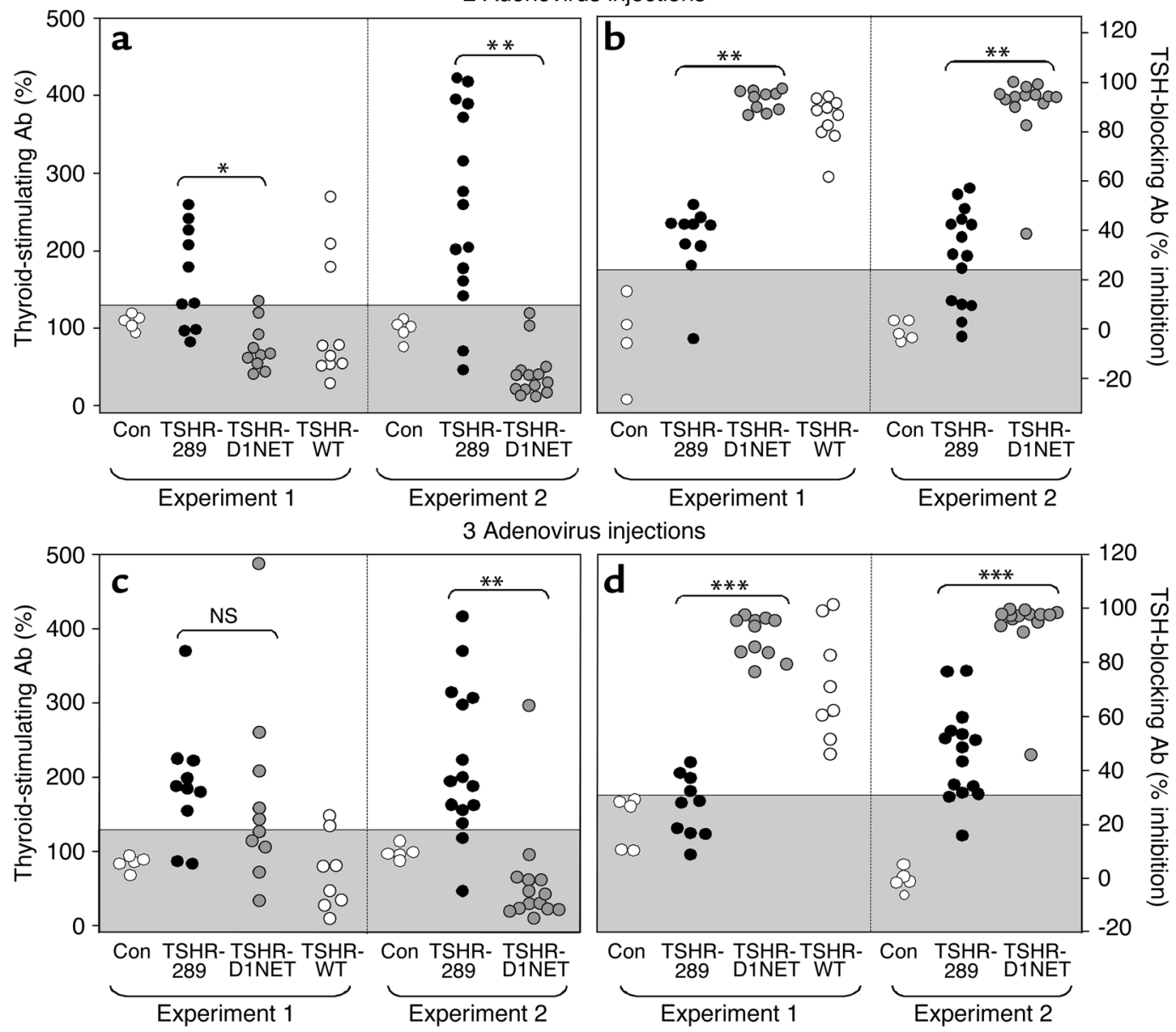

Figure 6

TSAb's and TBAb's in mice injected with adenovirus expressing different TSHR constructs. BALB/c mice were immunized intramuscularly with adenovirus expressing the shed A subunit (TSHR-289), the noncleaving receptor (TSHR-D1NET), the WT receptor (TSHR-WT), and control adenovirus (Con). Sera were studied after two injections ( $\mathbf{a}$ and $\mathbf{b}$ ) and at euthanasia after three injections (c and $\mathbf{d})$. Data for individual mice are presented as percentage of control sera for TSAb and as percentage of inhibition of TSH activity for TBAb. Absolute cAMP values for the control sera were $2.9 \pm 0.17 \mathrm{pmol} /$ well in the absence of TSH and $61.7 \pm 2.6 \mathrm{pmol} /$ well in the presence of $0.2 \mathrm{mU} / \mathrm{ml} \mathrm{TSH}$ (mean $\pm \mathrm{SE}, n=4)$. The gray area indicates the mean $\pm 2 \mathrm{SD}$ of values for 15 control adenovirus-immunized mice. Values significantly different between indicated groups are ${ }^{*} P=0.002 ;{ }^{*} P<0.002 ;{ }^{*}{ }^{*} P<0.001$ (Mann-Whitney rank sum test).

thyrotoxicosis also had the lowest TBAb activity to constrain their TSAb activity.

\section{Discussion}

The present data advance our understanding as to why autoantibodies arise to the TSHR (causing Graves hyperthyroidism) but not to the closely related gonadotrophin receptors. The propensity of the TSHR to shed part of its autoantibody-binding region has been known for many years $(24,25)$. More recently, the shed portion of the TSHR has been identified as the A subunit $(7,8)$. It has been suggested that a shed A subunit in serum may play a role in human autoimmunity or cancer $(7,26)$. There are no data demonstrating the presence of TSHR A subunits in serum, however, nor any other role for this fragment in disease pathogenesis. We now show, as summarized below, that rather than the TSH holoreceptor, the free TSHR A subunit is the primary immunogen in the development of a spectrum of TSHR autoantibodies that cause Graves disease.

Shedding of the TSHR A subunit can only occur after ectodomain cleavage. If stimulatory TSHR Ab's (TSAb's) arise primarily in response to the shed A subunit, immunization with adenovirus expressing a TSHR engineered not to cleave into subunits should be a poor inducer of Graves-like hyperthyroidism in mice. Conversely, injecting mice with adenovirus expressing the free A subunit should potentiate the development of hyperthyroidism. This hypothesis was, indeed, fulfilled, but with a caveat regarding the reciprocal roles of TSAb and TBAb (see below). Immunization with adenovirus expressing an $\mathrm{A}$ subunit produced goiter and thyrotoxicosis in the majority of animals. In contrast, goiter and thyrotoxicosis occurred in only a small proportion of animals receiving an adenovirus expressing a noncleaving TSHR. Intermediate values were observed for animals immunized with the WT TSHR. Because a significant and variable 
proportion of TSHRs on the cell surface do not undergo intramolecular cleavage into subunits (reviewed in ref. 2 ), the WT receptor (unlike TSHR-D1NET) is not an adequate control in these experiments.

An important consideration in evaluating our results is whether the different incidences of goiter and thyrotoxicosis among groups of animals simply reflect quantitative rather than qualitative differences in the immune response to the TSHR induced by the different adenovirus constructs. This possibility was excluded by TSHR-289 adenovirus immunization inducing quantitatively similar or lower TSHR Ab levels measured by ELISA (Figure 4) than with TSHR-D1NET adenovirus immunization. Moreover, TBI activities were also similar among the groups (Figure 5); TBI correlates more closely with quantitative TSHR Ab-binding than with Ab functional activity (27-29). Finally, the noncleaving TSHR that poorly induced goiter and thyrotoxicosis was the strongest immunogen for generating Ab's that blocked TSH-induced cAMP generation (TBAb) (Figure 6). Conversely, the A subunit-expressing adenovirus, highly effective in producing Graveslike hyperthyroidism, was the weakest inducer of TBAb. Whether immunization produced goiter and thyrotoxicosis, therefore, clearly depended on the qualitative, not quantitative, nature of the immune response.

Since the submission of our study, several murine mAb's with stimulatory activity (TSAb's) have been isolated from mice or hamsters immunized with the WT TSHR (30-32). In recent years, a variety of immunization approaches have been used to generate TSAb and hyperthyroidism in animals (for example, refs. 10-13, 31 ). Despite these achievements, it has been surprisingly difficult to generate monoclonal TSAb's from these animals. Our data provide insight into the reason for this difficulty. The WT TSHR is effective in generating TBAb, and its ability to stimulate TSAb may depend on the extent of cleavage and A subunit shedding in each of these experimental models.

TSHR autoantibodies, as measured by TBI or functional bioassays for TSAb, are detected in more than $90 \%$ of untreated Graves patients. Nevertheless, quantitatively, the correlation between these two assays is not very high, nor does TSHR Ab titer correlate closely with the severity of hyperthyroidism. Two explanations have been offered for these inconsistencies. First, TSAb-mediated hyperthyroidism may be dampened by a varying degree of autoimmune thyroiditis (so-called "hashitoxicosis"). Second, TSAb potency can be offset by fluctuating levels of TBAb, causing some patients to swing between hyperthyroidism and hypothyroidism (for example, see refs. 33, 34). Our study supports the importance of the latter phenomenon. We find that the free, or shed, A subunit induces TSAb but less TBAb. In contrast, the holoreceptor induces some TSAb, but is a much more potent immunogen for TBAb that can suppress the function of the former. These data indicate that, as in human Graves disease, measuring TSAb by itself is of limited value in evaluating the severity of disease. In our study, goiter size and thyroid hormone levels, not Ab's, were the "gold standard" in assessing the functional consequences of immunizing with different structural forms of the TSHR.

Other aspects of measuring TSAb in our study require discussion. When used to evaluate Graves disease in humans, the TSAb bioassay uses purified IgG to minimize the influence of other serum factors. IgG is then assayed at the original, very high serum concentration. The limited amount of serum available from individual mice precludes IgG purification. Assay of unseparated serum is possible, but only after serum dilution (1:33 in our study) at the expense of reduced sensitivity. It is, therefore, to be expected that the incidence of TSAb in immunized animals is lower in this in vitro assay than the occurrence of goiter and hyperthyroidism in vivo. Serum dilution also has a major effect on the detection of TSAb. In the presence of high TBAb activity, Zakarija and McKenzie observed that TSAb activity may emerge only after serum dilution or after the decline in neonatal TSHR Ab levels following transplacental passage from the mother (35).

Mice injected with Ad-TSHR-289 share several fundamental characteristics of Graves patients, including hyperthyroidism, goiter, TSAb, and the histological features of stimulated thyrocytes. One noteworthy difference compared with human disease, however, is the high level of TSHR Ab's on ELISA (detectable even when diluted 1:103) in the TSHR-adenovirus model. It is difficult to detect TSHR Ab's in Graves patients by direct-binding assays, and undiluted sera are usually required for their detection (27-29). Incidentally, it is unclear why the incidence of elevated serum T4 levels is lower in our animals immunized with the WT TSHR (particularly after the third injection) than in the original description of the adenovirus model for Graves disease (13). On the other hand, the incidence of goiter (approximately $50 \%$ ), a cardinal feature of thyroid stimulation, was similar in both studies.

Growing evidence suggests that cleavage of some proteins during apoptosis exposes autoantigenic epitopes that are cryptic in the uncleaved molecule. Examples of this phenomenon include a subset of nuclear proteins in systemic lupus erythematosus (36) and $\alpha$-fodrin in Sjogren syndrome (37). Unlike the related disorder, Hashimoto thyroiditis, Graves disease is not associated with marked cell death and apoptosis $(38,39)$. The mechanisms responsible for TSHR cleavage are unclear, but may involve matrix metalloproteinases (40). The TSHR, but not the luteinizing hormone receptor, accumulates in the regions of cell contact with the extracellular matrix, where putative cleavage enzymes also tend to concentrate (41). Variability of TSHR cleavage in vitro by cell culture conditions, for example enhancement by low fetal calf concentrations (8), raises the possibility that cleavage of the TSHR in vivo may vary in response to infections or possibly as a polymorphism between different individuals.

In summary, our data provide strong evidence that an unusual structural feature of the TSHR (spontaneous cleavage and release of the autoantibody-binding A subunit) plays a role in the development of goiter and 
hyperthyroidism in Graves disease. TSHR A subunits, far more than the holoreceptor, induce TSHR Ab's biased toward thyroid stimulation (TSAb with a very low proportion of TBAb). Shed A subunits are more likely to migrate to regional lymph nodes than to achieve a significant level in plasma. The unusually high glycan content (approximately $40 \%$ ) of the A subunit (14) increases the likelihood of uptake in draining lymph nodes by dendritic cells (primary antigen-presenting cells), perhaps via their mannose receptors (42). Remarkably, the autoantigen in Graves disease is the culprit as well as the victim, a finding with novel conceptual implications for autoimmune diseases in general.

\section{Acknowledgments}

We thank H. Mizuguchi (Division of Biological Chemistry and Biologicals, National Institute of Health Sciences, Tokyo, Japan) for providing the adenoviral vectors. This work was supported by NIH grants DK-54684 and DK-19289 and by the generous contributions of Boris Catz.

1. Vanderpump, M.P.J., et al. 1995. The incidence of thyroid disorders in the community: a twenty-year follow-up of the Whickham Survey. Clin. Endocrinol.(Oxf.). 43:55-68.

2. Rapoport, B., Chazenbalk, G.D., Jaume, J.C., and McLachlan, S.M. 1998 The thyrotropin receptor: interaction with thyrotropin and autoantibodies. Endocr. Rev. 19:673-716.

3. Tomer, Y., Barbesino, G., Greenberg, D.A., Concepcion, E., and Davies, T.F. 1999. Mapping the major susceptibility loci for familial Graves' and Hashimoto's diseases: evidence for genetic heterogeneity and gene interactions. J. Clin. Endocrinol. Metab. 84:4656-4664.

4. Brix, T.H., Kyvik, K.O., Christensen, K., and Hegedus, L. 2001. Evidence for a major role of heredity in Graves' disease: a population-based study of two Danish twin cohorts. J. Clin. Endocrinol. Metab. 86:930-934.

5. Buckland, P.R., Rickards, C.R., Howells, R.D., Jones, E.D., and Rees Smith, B. 1982. Photo-affinity labeling of the thyrotropin receptor. FEBS Lett. 145:245-249.

6. Loosfelt, H., et al. 1992. Two-subunit structure of the human thyrotropin receptor. Proc. Natl. Acad. Sci. U. S. A. 89:3765-3769.

7. Couet, J., et al. 1996. Shedding of human thyrotropin receptor ectodomain: involvement of a matrix metalloprotease. J. Biol. Chem. 271:4545-4552

8. Tanaka, K., Chazenbalk, G.D., McLachlan, S.M., and Rapoport, B. 1999. The shed component of the TSH receptor is primarily a carboxyl terminal truncated form of the A subunit, not the entire A subunit. Mol. Cell Endocrinol. 150:113-119.

9. Chazenbalk, G.D., et al. 2002. Thyroid-stimulating autoantibodies in Graves disease preferentially recognize the free A subunit, not the thyrotropin holoreceptor. J. Clin. Invest. 110:209-217. doi:10.1172/JCI200215745.

10. Shimojo, N., et al. 1996. Induction of Graves-like disease in mice by immunization with fibroblasts transfected with the thyrotropin receptor and a class II molecule. Proc. Natl. Acad. Sci. U. S. A. 93:11074-11079.

11. Costagliola, S., et al. 2000. Genetic immunization of outbred mice with thyrotropin receptor cDNA provides a model of Graves' disease. J. Clin. Invest. 105:803-811.

12. Kaithamana, S., Fan, J., Osuga, Y., Liang, S.G., and Prabhakar, B.S. 1999. Induction of experimental autoimmune Graves' disease in $\mathrm{BALB} / \mathrm{c}$ mice. J. Immunol. 163:5157-5164.

13. Nagayama, Y., et al. 2002. A novel murine model of Graves' hyperthyroidism with intramuscular injection of adenovirus expressing the thyrotropin receptor. J. Immunol. 168:2789-2794.

14. Chazenbalk, G.D., Jaume, J.C., McLachlan, S.M., and Rapoport, B. 1997. Engineering the human thyrotropin receptor ectodomain from a non-secreted form to a secreted, highly immunoreactive glycoprotein that neutralizes autoantibodies in Graves' patients' sera. J. Biol. Chem. 272:18959-18965

15. Tanaka, K., Chazenbalk, G.D., McLachlan, S.M., and Rapoport, B. 1998 Thyrotropin receptor cleavage at site 1 does not involve a specific amino acid motif but instead depends on the presence of the unique, 50 amino acid insertion. J. Biol. Chem. 273:1959-1963.

16. Mizuguchi, H., and Kay, M.A. 1998. Efficient construction of a recombinant adenovirus vector by an improved in vitro ligation method. Hum. Gene Ther. 9:2577-2583.
17. Mizuguchi, H., and Kay, M.A. 1999. A simple method for constructing E1- and E1/E4-deleted recombinant adenoviral vectors. Hum. Gene Ther. 10:2013-2017.

18. Mittereder, N., March, K.L., and Trapnell, B.C. 1996. Evaluation of the concentration and bioactivity of adenovirus vectors for gene therapy. J. Virol. 70:7498-7509.

19. Pichurin, P., et al. 2001. Naked thyrotropin receptor DNA vaccination: a TH1 $\mathrm{T}$ cell response in which interferon- $\gamma$ production, rather than antibody, dominates the immune response in mice. Endocrinology. 142:3530-3536

20. Chazenbalk, G.D., et al. 1999. A mouse monoclonal antibody to a thyrotropin receptor ectodomain variant provides insight into the exquisite antigenic conformational requirement, epitopes and in vivo concentration of human autoantibodies. J. Clin. Endocrinol. Metab. 84:702-710.

21. Kita, M., et al. 1999. Regulation and transfer of a murine model of thyrotropin receptor antibody mediated Graves' disease. Endocrinology. 140:1392-1398.

22. Pichurin, P., et al. 2002. Immune deviation away from Th1 in interferongamma knock-out mice does not enhance TSH receptor antibody production after naked DNA vaccination. Endocrinology. 143:1182-1189.

23. Kakinuma, A., Chazenbalk, G., Filetti, S., McLachlan, S.M., and Rapoport, B. 1996 . Both the $5^{\prime}$ and $3^{\prime}$ non-coding regions of the thyrotropin receptor messenger RNA influence the level of receptor protein expression in transfected mammalian cells. Endocrinology. 137:2664-2669.

24. Smith, B.R. 1970. The interaction of the long-acting thyroid stimulator (LATS) with thyroid tissue in vitro. J. Endocrinol. 46:45-54.

25. Foti, D., Russo, D., Costante, G., and Filetti, S. 1991. The biological activity of bovine and human thyrotropin is differently affected by trypsin treatment of human thyroid cells: thyroid-stimulating antibody is related to human thyrotropin. J. Clin. Endocrinol. Metab. 73:710-716.

26. Misrahi, M., and Milgrom, E. 1997. Cleavage and shedding of the TSH receptor. Eur. J. Endocrinol. 137:599-602.

27. Chazenbalk, G.D., Pichurin, P., McLachlan, S.M., and Rapoport, B. 1999 A direct binding assay for thyrotropin receptor autoantibodies. Thyroid. 9:1057-1061.

28. Sanders, J., et al. 1999. The interaction of TSH receptor autoantibodies with 125I-labeled TSH receptor. J. Clin. Endocrinol. Metab. 84:3797-3802.

29. Minich, W.B., and Loos, U. 1999. Isolation of radiochemically pure 125Ilabeled human thyrotropin receptor and its use for the detection of pathological autoantibodies in sera from Graves' patients. J. Endocrinol. 160:239-245

30. Sanders, J., et al. 2002. Thyroid stimulating monoclonal antibodies. Thyroid. 12:1043-1050.

31. Ando, T., Imaizumi, M., Graves, P., Unger, P., and Davies, T.F. 2003 Induction of thyroid-stimulating hormone receptor autoimmunity in hamsters. Endocrinology. 144:671-680.

32. Costagliola, S., et al. 2002. Generation of a mouse monoclonal TSH receptor antibody with stimulating activity. Biochem. Biophys. Res. Commun. 299:891-896.

33. Kraiem, Z., Baron, E., Kahana, L., Sadeh, O., and Sheinfeld, M. 1992. Changes in stimulating and blocking TSH receptor antibodies in a patient undergoing three cycles of transition from hypo to hyper-thyroidism and back to hypothyroidism. Clin. Endocrinol. (Oxf.). 36:211-214.

34. Kasagi, K., et al. 1993. Fluctuating thyroid function depending on the balance between stimulating and blocking types of TSH receptor antibodies: a case report. Thyroid. 3:315-318.

35. Zakarija, M., McKenzie, J.M., and Munro, D.S. 1983. Immunoglobulin $\mathrm{G}$ inhibitor of thyroid-stimulating antibody is a cause of delay in the onset of neonatal Graves' disease. J. Clin. Invest. 72:1352-1356.

36. Casiano, C.A., Martin, S.J., Green, D.R., and Tan, E.M. 1996. Selective cleavage of nuclear autoantigens during CD95 (Fas/APO-1)-mediated T cell apoptosis. J. Exp. Med. 184:765-770.

37. Saegusa, K., et al. 2002. Prevention and induction of autoimmune exocrinopathy is dependent on pathogenic autoantigen cleavage in murine Sjogren's syndrome. J. Immunol. 169:1050-1057.

38. Hammond, L.J., et al. 1997. Analysis of apoptosis in relation to tissue destruction associated with Hashimoto's autoimmune thyroiditis. J. Pathol. 182:138-144.

39. Salmaso, C., et al. 2002. Regulation of apoptosis in endocrine autoimmunity: insights from Hashimoto's thyroiditis and Graves' disease. Ann. N. Y. Acad. Sci. 966:496-501.

40. Couet, J., et al. 1996. Cell surface protein disulfide-isomerase is involved in the shedding of human thyrotropin receptor ectodomain. Biochemistry. 35:14800-14805.

41. Ghinea, N., Baratti-Elbaz, C., Jesus-Lucas, A., and Milgrom, E. 2002. TSH receptor interaction with the extracellular matrix: role on constitutive activity and sensitivity to hormonal stimulation. Mol. Endocrinol. 16:912-923.

42. Linehan, S.A., Martinez-Pomares, L., da Silva, R.P., and Gordon, S. 2001. Endogenous ligands of carbohydrate recognition domains of the mannose receptor in murine macrophages, endothelial cells and secretory cells; potential relevance to inflammation and immunity. Eur. J. Immunol. 31:1857-1866. 